\title{
17. ON THE ORIGIN OF WHITE DWARFS
}

\author{
B. PACZYŃSKI* \\ Institute of Theoretical Astronomy, University of Cambridge
}

\begin{abstract}
Model evolutionary calculations have been made for population I stars $(X=0.7$, $Z=0.03$ ) with masses of $0.8,1.5,3,5,7,10$, and $15 M_{\odot}$ (Paczyński, 1970). Neutrino losses were taken into account. All the models were evolved up to the red supergiant phase with the hydrogen and helium burning shell sources. In this phase of evolution the hydrogen rich envelopes of $0.8,1.5$, and $3 M_{\odot}$ stars became dynamically unstable due to the large depth of the hydrogen and helium ionization zones. It was assumed that almost entire envelopes were lost as a result of that instability. The degenerate carbon-oxygen cores of $0.6,0.8$, and $1.2 M_{\odot}$ were left with the hydrogen rich envelopes of a small mass. Almost all the hydrogen that was left was subsequently burnt in the shell source. Finally, the hydrogen and helium burning shell sources disappeared as the nuclear fuel was exhausted. The models were cooling down to the white dwarf phase and they had a small amount of hydrogen left close to their surfaces. It is estimated that population I stars with masses up to $3.5 M_{\odot}$ may produce white dwarfs with masses up to $1.37 M_{\odot}$. The hydrogen rich envelopes that were lost as a result of dynamical instability could form planetary nebulae with masses up to $2 M_{\odot}$. Further details are published in the paper referred to above.
\end{abstract}

\section{Reference}

Paczyński, B.: 1970, Acta Astron. $20,47$.

* On leave from the Institute of Astronomy, Polish Academy of Sciences. 